\title{
A FAMILY OF DOMINANT FITTING CLASSES OF FINITE SOLUBLE GROUPS
}

\section{A. BALLESTER-BOLINCHES, A. MARTÍNEZ-PASTOR and M. D. PÉREZ-RAMOS}

(Received 25 July 1996)

Communicated by R. B. Howlett

\begin{abstract}
In this paper a large family of dominant Fitting classes of finite soluble groups and the description of the corresponding injectors are obtained. Classical constructions of nilpotent and Lockett injectors as well as $p$-nilpotent injectors arise as particular cases.
\end{abstract}

1994 Mathematics subject classification (Amer. Math. Soc.): primary 20D10.

\section{Introduction}

Unless otherwise stated all groups considered in this paper are finite and soluble.

Although theories of formations and Fitting classes are quite independent generalizations of the classical theory of Sylow and Hall, many of their results have been motivated by the good behaviour of the Fitting formation of nilpotent groups as a class of groups. In the references [1-4] some extensions of nilpotent groups have been studied mainly within the framework of formation theory.

In [2], formations of groups which are the direct product of Hall subgroups corresponding to pairwise disjoint sets of primes appear. These formations were also studied by Lockett [6] as Fitting classes. He proves that they are dominant Fitting classes by giving a description of the injectors as generalization of the classical construction of the nilpotent ones. In [4], the formations $\mathscr{F}$ whose minimal non- $\mathscr{F}$-groups are Schmidt groups (that is, minimal non-nilpotent groups) are described. In [3], we met formations characterized through the existence of normal $\pi(p)$-complements in the groups of the class, for every prime $p$, where $\pi(p)$ is a set of primes containing $p$.

The common property of these formations $\mathscr{F}$ considered in the above references

(C) 1998 Australian Mathematical Society 0263-6115/98 \$A2.00+0.00 
is that they are saturated formations which are locally defined by $\mathscr{S}_{\pi(p)}$, the class of all $\pi(p)$-groups for a set of primes $\pi(p)$, for every prime $p$ in the characteristic of $\mathscr{F}$ and $p \in \pi(p)$. These different extensions of nilpotent groups arise by requiring restrictions on the sets of primes $\pi(p)$. Here we want to draw attention to the fact that they are also Fitting classes. So viewed, the results of the present paper provide a characterization for this type of Fitting classes to be dominant. A well-known result of Lockett [5, I.7.8] about the permutability of normally embedded subgroups of a group into which a given Hall system of the group reduces allows us to give the exact description of the injectors for these classes. Classical constructions of nilpotent injectors, Lockett's injectors, as well as $p$-nilpotent injectors ( $p$ a prime), appear as particular cases of our construction.

Again the above characterization rest on the appropriate restrictions for the sets of primes $\pi(p)$. This leads to a nice relation between dominance as Fitting classes and the description of the canonical local definition as saturated formations.

\section{Preliminary results; notation}

The notation is standard and is taken mainly from [5]. We also refer to this book for details about Fitting classes and formations. For completeness, we gather some notation and recall some definitions and results.

A Fitting class $\mathscr{F}$ is said to be dominant in $\mathscr{S}$, the class of all soluble groups, if $\mathscr{F} \subseteq \mathscr{S}$ and for every group $G \in \mathscr{S}$ any two $\mathscr{F}$-maximal subgroups of $G$ containing the $\mathscr{F}$-radical, $G_{\mathscr{F}}$, of $G$ are conjugate in $G$.

The following properties of dominant Fitting classes are used.

LEMMA 2.1 [5, IX.4.2]. Let $\mathscr{F}$ be a Fitting class which is dominant in $\mathscr{S}$. Then every group $G$ in $\mathscr{S}$ has a unique conjugacy class of $\mathscr{F}$-injectors, namely the $\mathscr{F}$ maximal subgroups of $G$ containing $G_{\mathscr{F}}$.

We denote by $\wp$ the set of all prime numbers and, for a prime $p, C_{p}$ as the cyclic group of order $p$. If $\mathscr{H}$ is a class of groups, the characteristic of $\mathscr{H}$ is char $\mathscr{H}=\left\{p \in \wp \mid C_{p} \in \mathscr{H}\right\}$. For a group $G, \pi(G)$ denotes the set of all prime divisors of $|G|$.

Proposition 2.1 [5, IX.4.3]. Let $\mathscr{F}$ be a Fitting class which is dominant in $\mathscr{S}$. Then either $\mathscr{N} \subseteq \mathscr{F}$ or $\mathscr{F}=\mathscr{S}_{\pi}$ for some $\pi \subseteq \wp$.

The well-known Gaschütz-Lubeseder-Schmid Theorem states that in the general finite universe, saturated formations are exactly local formations, that is, formations $\mathscr{F}=L F(f)$ defined by a formation function $f: L F(f)=(G \in \mathscr{E} \mid$ if $H / K$ is 
a chief factor of $G$ and $p \in \pi(H / K)$, then $\left.G / C_{G}(H / K) \in f(p)\right)$, where $\mathscr{E}$ is the class of all finite groups. In this case, $f$ is said to be a local definition of $\mathscr{F}$. Among all possible local definitions of a local formation $\mathscr{F}$ there exists exactly one, denoted by $F$, such that $F$ is integrated (that is, $F(p) \subseteq \mathscr{F}$ for all $p \in \wp$ ) and full (that is, $\mathscr{S}_{p} F(p)=F(p)$ for all $\left.p \in \wp\right)$, that is, $F$ is the canonical local definition of $\mathscr{F}$.

PROPOSITION 2.2 [5, IV.3.14]. If $f(p)$ is a subgroup-closed Fitting formation for all $p \in \wp$, then $\mathscr{F}=L F(f)$ is also a subgroup-closed Fitting formation.

We also state some results on $f$-hypercentral action.

DEFINITION [5, IV.6.2]. Let $A \in \mathscr{E}$ be a group of operators of the group $G \in$ $\mathscr{E}$. Given a formation function $f$, we say that $A$ acts $f$-hypercentrally on $G$ if $A / C_{A}(H / K) \in f(p)$ for all primes $p$ dividing $|H / K|$ and for every $A$-composition factor $H / K$ of $G$.

Lemma 2.2 [5, IV.6.4(c)]. Let $f$ be a formation function, let $G$ be an A-group, and let $M$ and $N$ be $A$-invariant normal subgroups of $G$. If $A$ acts $f$-hypercentrally on $M$ and $N$, then it acts similarly on $M N$.

THEOREM 2.1 [5, IV.6.9]. Let $\mathscr{F}=L F(f)$ with $f$ an integrated local definition of $\mathscr{F}$. If $A$ acts $f$-hypercentrally on a group $G$, then $A / C_{A}(G) \in \mathscr{F}$.

In the following, all groups considered are finite and soluble. Moreover we say that a Fitting class $\mathscr{F}$ is dominant if $\mathscr{F}$ is a Fitting class which is dominant in $\mathscr{S}$.

\section{The results}

In this section, $\mathscr{F}=L F(f)$ will be a saturated formation which is locally defined by the formation function $f$ given by $f(p)=\mathscr{S}_{\pi(p)}$, for a set of primes $\pi(p)$ such that $p \in \pi(p)$, for every prime $p$ in the characteristic of $\mathscr{F}$. By Proposition 2.2, $\mathscr{F}$ is also a Fitting class. Since our purpose is to characterize when $\mathscr{F}$ is dominant, we will assume by Proposition 2.1 that $\mathscr{F}$ is of full characteristic.

PROPOSITION 3.1. Assume that $\mathscr{F}$ is a dominant Fitting class. Then $\mathscr{F}$ has the following property:

$(*)$ If $q$ is a prime in $\pi(p)$, then either $\pi(q)=\pi(p)$ or $\pi(q)=\wp$ or $\pi(p)=\wp$.

PROOF. Assume that $q$ is a prime in $\pi(p)$ such that $\pi(p) \neq \wp$ and $\pi(q) \neq \wp$. We prove that $\pi(q)=\pi(p)$.

Suppose, arguing by contradiction, that $\pi(q)$ is not contained in $\pi(p)$. Let $r$ be a prime in $\pi(q) \backslash \pi(p)$. We distinguish two cases: 
Case 1: $p \in \pi(q)$. Since $p \in \pi(p)$, it is clear that $r \neq p$. Let $V_{p}$ be an irreducible and faithful $C_{r}$-module over $G F(p)$. Denote by $X=\left[V_{p}\right] C_{r}$ the corresponding semidirect product. Let $t$ be a prime such that $t \notin \pi(q)$. Since $t \neq p$, we can take an irreducible and faithful $X$-module $V_{t}$ over $G F(t)$. Consider $Y=\left[V_{t}\right] X$ to be the corresponding semidirect product. Since $t \neq q$, there exists an irreducible and faithful $Y$-module $V_{q}$ over $G F(q)$. Let $G$ denote $\left[V_{q}\right] Y$. It is clear that $G_{\mathscr{F}}$, the $\mathscr{F}$-radical of $G$, is exactly $V_{q}$. Therefore $V_{q} V_{p}$ and $V_{q} C_{r}$ are two non-conjugate $\mathscr{F}$-maximal subgroups of $G$ containing $G_{\mathscr{F}}$. This contradicts the dominance of $\mathscr{F}$.

Case $2 p \notin \pi(q)$. We argue as in the above case by constructing the group $G=\left[V_{p}\right]\left(\left[V_{r}\right]\left(\left[V_{q}\right] C_{p}\right)\right)$.

Consequently, $\pi(q) \subseteq \pi(p)$.

Now if there exists a prime $r \in \pi(p)$ such that $r \notin \pi(q)$, then we can construct the group $G=\left[V_{p}\right]\left(\left[V_{t}\right]\left(\left[V_{q}\right] C_{r}\right)\right)$, where $t \notin \pi(p)$. It is clear that $G$ violates the dominance of $\mathscr{F}$.

Our goal is to prove that the converse of the above proposition holds. So, in the sequel, $\mathscr{F}$ will always denote a saturated formation of full characteristic which is locally defined by the formation function $f$ given by $f(p)=\mathscr{S}_{\pi(p)}$, for a set of primes $\pi(p)$ such that $p \in \pi(p)$, for all $p \in \wp$. Moreover we shall assume that $\mathscr{F}$ satisfies the following condition:

If $q$ is a prime in $\pi(p)$, then either $\pi(q)=\pi(p)$ or $\pi(q)=\wp$ or $\pi(p)=\wp$, for every pair of primes $p, q \in \wp$.

First of all, we need to get information about $\mathscr{F}$ and the structure of its groups. Set $\pi=\{p \in \wp \mid \pi(p) \neq \wp\}$.

LEMMA 3.1. Consider the following equivalence relation on $\pi$ :

$$
p \sim q \quad \text { if and only if } \pi(p)=\pi(q), \quad p, q \in \pi .
$$

If $p, q \in \pi$ and $\pi(p) \neq \pi(q)$, then $\pi(p) \cap \pi(q) \subseteq \pi^{\prime}$. Consequently, the equivalence class of an element $p \in \pi$ is $\pi(p) \cap \pi$. In particular, if $\hat{\pi}$ is a representative complete system, it is $\pi=\bigcup_{p \in \hat{\pi}}(\pi(p) \cap \pi)$.

PROOF. Follows from the above.

LEMMA 3.2. If $p \in \pi$, then $\mathscr{S}_{\pi(p)} \subseteq \mathscr{F}$. Consequently, $f(p)=F(p)$, for every prime $p \in \pi$, where $F$ is the canonical local definition of $\mathscr{F}$. 
ProOF. Assume the result is not true and let $G \in \mathscr{S}_{\pi(p)} \backslash \mathscr{F}$ be a group of minimal order. $G$ has a unique minimal normal subgroup, $N$ say, such that $N=C_{G}(N)$, $G / N \in \mathscr{F}$ and $N$ is a $q$-group for some prime $q \in \pi(p)$. Since either $\pi(q)=\pi(p)$ or $\pi(q)=\wp$, we have that $G / C_{G}(N) \in f(q)$. This implies that $G \in \mathscr{F}$, a contradiction.

In fact, the property stated in Lemma 3.2 is equivalent to condition (*). Notice that this provides a description of the canonical local definition of $\mathscr{F}$. More precisely, we have the following:

PROPOSITION 3.2. Let $\mathscr{H}$ be a saturated formation which is locally defined by the formation function $h$ given by $h(p)=\mathscr{S}_{\pi(p)}$, where $\pi(p)$ is a set of primes with $p \in \pi(p) \subseteq$ char $\mathscr{H}$, for all $p \in$ char $\mathscr{H}$. The following statements are pairwise equivalent:

(i) If $q \in \pi(p)$, then either $\pi(q)=\pi(p)$ or $\pi(q)=\wp$ or $\pi(p)=\wp$.

(ii) If $q \in \pi(p) \neq \wp$, then $\pi(p) \subseteq \pi(q)$.

(iii) If $\pi(p) \neq \wp$, then $\mathscr{S}_{\pi(p)} \subseteq \mathscr{H}$.

(iv) The canonical local definition of $\mathscr{H}$ is given by

$H(p)=h(p)=\mathscr{S}_{\pi(p)}$, for every prime $p \in$ char $\mathscr{H}$ such that $\pi(p) \neq \wp$, $H(q)=\mathscr{H}$, for every prime $q \in \operatorname{char} \mathscr{H}$ such that $\pi(q)=\wp$.

ProOF. That (i) implies (iii) follows as Lemma 3.2. For (iii) implies (ii), assume that $q \in \pi(p) \neq \wp$ and let $r \in \pi(p)$. If $r \neq q$ and $V_{q}$ is an irreducible and faithful $C_{r}$-module over $G F(q)$, then $\left[V_{q}\right] C_{r} \in \mathscr{S}_{\pi(p)} \subseteq \mathscr{H}$ by hypothesis. Hence $C_{r} \in h(q)$ and $r \in \pi(q)$.

We now show (ii) implies (i): Assume that $q \in \pi(p), \pi(p) \neq \wp$ and $\pi(q) \neq \wp$. By hypothesis, we have $\pi(p) \subseteq \pi(q)$. Since $p \in \pi(p)$, we also have $\pi(q) \subseteq \pi(p)$.

The equivalence between (iii) and (iv) is clear.

LEMMA 3.3. $\mathscr{S}_{\pi}, \mathscr{F}=\mathscr{F}$.

PROOF. This follows directly from the fact that if $r \in \pi^{\prime}$ then $\pi(r)=\wp$, so $f(r)=\mathscr{S}$.

LEMMA 3.4. $\mathscr{F}$ is the smallest saturated formation satisfying $\mathscr{S}_{\pi^{\prime}} \mathscr{F}=\mathscr{F}$ and $\mathscr{S}_{\pi(p)} \subseteq \mathscr{F}$ for every $p \in \pi$.

PROOF. $\mathscr{F}$ is a saturated formation satisfying these properties by Lemmas 3.2 and 3.3.

Let $\mathscr{G}$ be a saturated formation such that $\mathscr{S}_{\pi} \mathscr{G}=\mathscr{G}$ and $\mathscr{S}_{\pi(p)} \subseteq \mathscr{G}$ for every $p \in \pi$. We claim that $\mathscr{F} \subseteq \mathscr{G}$. Assume this is not true and let $G \in \mathscr{F} \backslash \mathscr{G}$ be 
of minimal order. Then $G$ has a unique minimal normal subgroup $N$ which is a $p$-group for some prime $p, N=C_{G}(N)$, and $G / N \in \mathscr{G}$. It is clear that $p \in \pi$ since $\mathscr{S}_{\pi^{\prime}} \mathscr{G}=\mathscr{G}$. Then $G \in \mathscr{S}_{\pi(p)} \subseteq \mathscr{G}$, a contradiction.

We introduce some more notation.

Let $\hat{\pi}$ denote a representative complete system as in Lemma 3.1. For every prime $p \in \pi$, let

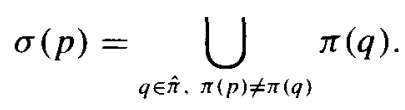

PROPOSITION 3.3. $\mathscr{F}=\mathscr{S}_{\pi^{\prime}}\left[\bigcap_{p \in \hat{\pi}}\left(\mathscr{S}_{\sigma(p)} \mathscr{S}_{\pi(p)}\right)\right]=\bigcap_{p \in \hat{\pi}}\left(\mathscr{S}_{\pi^{\prime}} \mathscr{S}_{\sigma(p)} \mathscr{S}_{\pi(p)}\right)$.

PROOF. Define $\mathscr{L}_{1}=\mathscr{S}_{\pi^{\prime}}\left[\bigcap_{p \in \hat{\pi}}\left(\mathscr{S}_{\sigma(p)} \mathscr{S}_{\pi(p)}\right)\right]$ and $\mathscr{L}_{2}=\bigcap_{p \in \hat{\pi}}\left(\mathscr{S}_{\pi^{\prime}} \mathscr{S}_{\sigma(p)} \mathscr{S}_{\pi(p)}\right)$. We claim that $\mathscr{F}=\mathscr{L}_{1}$. From Lemma 3.4 it is clear that $\mathscr{F} \subseteq \mathscr{L}_{1}$. Suppose that $\mathscr{F} \neq \mathscr{L}_{1}$ and take $G \in \mathscr{L}_{1} \backslash \mathscr{F}$ of minimal order. Then $G$ has a unique minimal normal subgroup $N$ such that $G / N \in \mathscr{F}$. If $N \in \mathscr{S}_{\pi^{\prime}}$, then $G \in \mathscr{S}_{\pi^{\prime}} \mathscr{F}=\mathscr{F}$ by Lemma 3.3, a contradiction. Consequently $N \in \mathscr{S}_{p}$, for some prime $p \in \pi$. Since $G \in \mathscr{L}_{1}$, we have that $O^{\pi(p)}(G) \in \mathscr{S}_{\sigma(p)}$. Moreover, $O^{\pi(p)}(G) \neq 1$ because $\mathscr{S}_{\pi(p)} \subseteq \mathscr{F}$ by Lemma 3.2. Therefore $N \leq O^{\pi(p)}(G) \in \mathscr{S}_{\sigma(p)}$. This implies $p \in \sigma(p)=\bigcup_{q \in \hat{\pi} \cdot \pi(p) \neq \pi(q)} \pi(q)$, a contradiction.

On the other hand, it is clear that $\mathscr{L}_{1} \subseteq \mathscr{L}_{2}$. The converse follows by a standard argument.

COROLLARY 3.1. For every group $G$ the $\mathscr{F}$-radical of $G$ is

$$
G_{\mathscr{F}}=\bigcap_{p \in \hat{\pi}} O_{\pi^{\prime} \sigma(p) \pi(p)}(G) .
$$

Next we provide the construction of suitable $\mathscr{F}$-injectors for the Fitting formations in consideration. The goal is to prove that these subgroups constitute a unique conjugacy class of $\mathscr{F}$-maximal subgroups containing $G_{\mathscr{F}}$ in every group $G$.

Notation and construction. For each prime $s$, consider the following partition of the set of primes $\pi$ :

$$
\pi=\left\{p_{i} \mid i \in I\right\} \bigcup\left\{p_{j} \mid j \in J\right\}
$$

in such manner that $s \notin \bigcup_{i \in l} \pi\left(p_{i}\right)$ but $s \in \bigcap_{j \in J} \pi\left(p_{j}\right)$.

For a group $G$, define

$$
\begin{aligned}
Z_{s}=\bigcap\left\{C_{G}(A / B) \mid A / B \text { runs over the chief factors of } G\right. \\
\text { between } \left.O_{\pi^{\prime}}(G) \text { and } O_{\pi^{\prime} r}(G) \text { for every } r \in\left\{p_{i} \mid i \in I\right\}\right\} .
\end{aligned}
$$


Use the usual convention that $Z_{s}=G$ if there does not exist such chief factors.

Take $\Sigma$ a Hall system of the group $G$. For every prime $s$, consider $H_{s}=G_{s} \cap Z_{s}$, for $G_{s} \in \operatorname{Syl}_{s}(G)$ such that $G_{s} \in \Sigma$. Since $Z_{s}$ is a normal subgroup of $G$, it is clear that $H_{s} \in \operatorname{Syl}_{s}\left(Z_{s}\right)$. Hence $H_{s}$ is a normally embedded subgroup of $G$ into which the Hall system $\Sigma$ reduces. A well-known result of Lockett [5, Th. I.7.8] tells us that $H_{s} H_{t}=H_{t} H_{s}$ and $H_{s} H_{t}$ is a normally embedded subgroup of $G$ into which $\Sigma$ reduces, for every pair of primes $t, s$. Consequently $H_{\Sigma}:=\prod_{s \in \pi(G)} H_{s}$ is a subgroup of $G$ with pairwise permutable factors. Notice that for every $s \in \pi(G), H_{s} \in \operatorname{Syl}_{s}\left(H_{\Sigma}\right), H_{\Sigma}$ is a normally embedded subgroup of $G$ and $\Sigma$ reduces into $H_{\Sigma}$.

For a group $G, Y_{G}=\prod_{p \in \pi} O_{\pi^{\prime} p}(G)$. Notice that $Y_{G}$ is contained in $G_{\mathscr{F}}$. Moreover $Y_{G} / O_{\pi^{\prime}}(G)=F\left(G / O_{\pi^{\prime}}(G)\right)$ and, consequently, $C_{G}\left(Y_{G} / O_{\pi^{\prime}}(G)\right) \leq Y_{G}$.

In the following, we consider a fixed arbitrary group $G$ for which the above notation is assumed.

REMARK. In order to construct the subgroups $H_{\Sigma}$ we can also consider the following subgroup instead of $Z_{s}$ :

$$
\begin{aligned}
Z_{s}^{*}: & =\bigcap_{r \in\left\{p_{i} \mid i \in I\right\}} C_{G}\left(O_{\pi^{\prime} r}(G) / O_{\pi^{\prime}}(G)\right) \\
& =C_{G}\left(\prod_{r \in\left\{p_{i} \mid i \in I\right\}} O_{\pi^{\prime} r}(G) / O_{\pi^{\prime}(G)}\right)
\end{aligned}
$$

for each prime $s$. This is because a standard argument of coprime action allows us to state that $\operatorname{Syl}_{s}\left(Z_{s}^{*}\right)=\operatorname{Syl}_{s}\left(Z_{s}\right)$, for every prime $s$. In particular, $H_{s}=G_{s} \cap Z_{s}=$ $G_{s} \cap Z_{s}^{*}$, for every prime $s$.

The properties of the subgroups $H_{\Sigma}$ will be developed in the following lemmas:

LEMMA 3.5. Let $L$ be a subgroup of $G$ such that $Y_{G}$ is contained in $L$. If $L$ belongs to $\mathscr{F}$ and $\Sigma$ is a Hall system of $G$ reducing into $L$, then $L$ is contained in $H_{\Sigma}$. In particular, $G_{\mathscr{F}}$ contained in $H_{\Sigma}$.

PROOF. By construction of $H_{\Sigma}$, it is enough to prove that $L_{s}:=L \cap G_{s}$ is contained in $Z_{s}$ for every prime $s \in \pi(G)$.

Let $s \in \pi(G)$ and $A / B$ be a chief factor of $G$ between $O_{\pi^{\prime}}(G)$ and $O_{\pi^{\prime} r}(G)$ for a prime $r \in\left\{p_{i} \mid i \in I\right\}$. Notice that $B \leq A \leq O_{\pi^{\prime} r}(G) \leq Y_{G}$. Consider

$$
1 \unlhd \cdots \unlhd B=B_{0} \unlhd \cdots \unlhd B_{i} \unlhd B_{i+1} \unlhd \cdots \unlhd B_{n}=A \unlhd \cdots \unlhd Y_{G} \unlhd \cdots \unlhd L,
$$

a chief series of $L$ through $B$ and $A$.

Since $L \in \mathscr{F}$, it follows that $L / C_{L}\left(B_{i+1} / B_{i}\right) \in \mathscr{S}_{\pi(r)}$, for every $0 \leq i \leq n-1$. The fact $s \notin \pi(r)$ implies that $L_{s} \leq C_{L}\left(B_{i+1} / B_{i}\right)$ for all $i \in\{0, \ldots, n-1\}$. This 
means that $L_{s}$ centralizes $A / B$ by $\left[5\right.$, A.12.3]. Hence $L_{s}$ is contained in $Z_{s}$ and the result follows.

LEMMA 3.6. $H_{\Sigma}$ acts $F$-hypercentrally on $Y_{G} / O_{\pi^{\prime}}(G)$. Consequently, $\left(H_{\Sigma}\right)^{\mathscr{F}}$ is contained in $C_{H_{\Sigma}}\left(Y_{G} / O_{\pi^{\prime}}(G)\right)$.

Proof. By Lemma 2.2 and the definition of $Y_{G}$, it is enough to prove that $H_{\Sigma}$ acts $F$-hypercentrally on $O_{\pi^{\prime} p}(G) / O_{\pi^{\prime}}(G)$ for every $p \in \pi$.

For each prime $p \in \pi$, consider

$$
1 \unlhd \cdots \unlhd O_{\pi^{\prime}}(G) \unlhd \cdots \unlhd B \unlhd A \unlhd \cdots \unlhd O_{\pi^{\prime} p}(G) \unlhd \cdots \unlhd G,
$$

a chief series of $G$ through $O_{\pi^{\prime}}(G)$ and $O_{\pi^{\prime} p}(G)$. Take $A / B$ to be a chief factor of this series between $O_{\pi^{\prime}}(G)$ and $O_{\pi^{\prime} p}(G)$. By construction of $H_{\Sigma}$, if $s \notin \pi(p)$, then $H_{s}$ is contained in $C_{H_{\Sigma}}(A / B)$. Therefore $H_{\Sigma} / C_{H_{\Sigma}}(A / B) \in \mathscr{S}_{\pi(p)}=f(p)=F(p)$ by Lemma 3.2.

Refine the above series to an $H_{\Sigma}$-composition series

$$
\begin{array}{r}
\cdots \unlhd O_{\pi^{\prime}}(G) \unlhd \cdots \unlhd B=B_{0} \unlhd \cdots \unlhd B_{i} \unlhd B_{i+1} \unlhd \cdots \\
\cdots \unlhd B_{n}=A \unlhd \cdots \unlhd O_{\pi^{\prime} p}(G) \unlhd \cdots
\end{array}
$$

Clearly $C_{H_{\Sigma}}(A / B)$ is contained in $C_{H_{\Sigma}}\left(B_{i+1} / B_{i}\right)$, for all $0 \leq i \leq n-1$. This implies that $H_{\Sigma} / C_{H_{\Sigma}}\left(B_{i+1} / B_{i}\right) \in \mathscr{S}_{\pi(p)}=F(p)$, for all $0 \leq i \leq n-1$. Consequently $H_{\Sigma}$ acts $F$-hypercentrally on $O_{\pi^{\prime} p}(G) / O_{\pi^{\prime}}(G)$.

Since $F$ is an integrated local definition of $\mathscr{F}$, by Theorem 2.1 it follows $H_{\Sigma} / C_{H_{\Sigma}}\left(Y_{G} / O_{\pi^{\prime}}(G)\right) \in \mathscr{F}$. Hence $\left(H_{\Sigma}\right)^{\mathscr{F}}$ is contained in $C_{H_{\Sigma}}\left(Y_{G} / O_{\pi^{\prime}}(G)\right)$.

LEMMA 3.7. $H_{\Sigma}$ belongs to $\mathscr{F}$.

ProOF. Notice that $O_{\pi^{\prime}}(G) \leq Y_{G} \leq G_{\mathscr{F}} \leq H_{\Sigma}$ by Lemma 3.5. By Lemma 3.6, $\left(H_{\Sigma}\right)^{\mathscr{F}}$ centralizes $Y_{G} / O_{\pi^{\prime}}(G)$. Hence $\left(H_{\Sigma}\right)^{\mathscr{F}}$ is contained in $Y_{G}$. Since $H_{\Sigma}$ acts $F$-hypercentrally on $Y_{G} / O_{\pi^{\prime}}(G)$, it follows that $H_{\Sigma} / O_{\pi^{\prime}}(G) \in \mathscr{F}$. So $H_{\Sigma} \in \mathscr{F}$ by Lemma 3.3 and the result is proved.

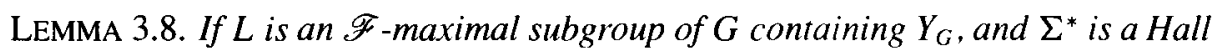
system of $G$ reducing into $L$, then $H_{\Sigma^{*}}=L$.

Proof. By Lemma 3.5, $L$ is contained in $H_{\Sigma^{*}}$, which belongs to $\mathscr{F}$ by the above lemma. Therefore $L=H_{\Sigma}$, by $\mathscr{F}$-maximality of $L$. 
LEMMA 3.9. For every Hall system $\Sigma$ of $G, H_{\Sigma}$ is an $\mathscr{F}$-maximal subgroup of $G$ containing $G_{\mathscr{F}}$.

PROOF. Notice firstly that $H_{\Sigma^{k}}=\left(H_{\Sigma}\right)^{g}$ for every $g \in G$. From Lemmas 3.5 and 3.7, we know that $H_{\Sigma}$ is an $\mathscr{F}$-subgroup of $G$ containing $G_{\mathscr{F}}$. Let $L$ be an $\mathscr{F}$-maximal subgroup of $G$ containing $H_{\Sigma}$ and let $\Sigma_{L}$ be a Hall system of $L$ reducing into $H_{\Sigma}$. Take now a Hall system $\Sigma^{*}$ of $G$ such that $\Sigma_{L}=\Sigma^{*} \cap L$. By [5, I.4.11], we can find an element $g \in G$ such that $\Sigma^{*}=\Sigma^{g}$. Since the Hall systems $\Sigma^{*}$ and $\Sigma$ of $G$ reduce into $H_{\Sigma}$ and $H_{\Sigma}$ is a pronormal subgroup of $G$, we have that $H_{\Sigma}=H_{\Sigma}$ * by [5, I.6.6]. Therefore $L=H_{\Sigma}{ }^{*}=H_{\Sigma}$ by Lemma 3.5. Hence $H_{\Sigma}$ is an $\mathscr{F}$-maximal subgroup of $G$ and the result is proved.

Now we can state the following

THEOREM 3.1. The set of $\mathscr{F}$-maximal subgroups of $G$ containing $G_{\mathscr{F}}$ is $\left\{H_{\Sigma} \mid \Sigma\right.$ is a Hall system of $G$ \}.

Notice that this is exactly the set of $\mathscr{F}$-maximal subgroups of $G$ containing $Y_{G}$.

Since the group $G$ acts transitively by conjugation on the set of its Hall systems, we have:

THEOREM 3.2. If $\Sigma_{1}$ and $\Sigma_{2}$ are two Hall systems of $G$, then $H_{\Sigma_{1}}$ and $H_{\Sigma_{2}}$ are conjugate. In particular there exists a unique $G$-conjugacy class of $\mathscr{F}$-maximal subgroups of $G$ containing $G_{\mathscr{F}}$.

COROLlaRY 3.2. $\mathscr{F}$ is a dominant Fitting class. The $\mathscr{F}$-injectors of $G$ are exactly the subgroups $H_{\Sigma}$, for every Hall system $\Sigma$ of $G$.

Now we can state the following theorem which completes Proposition 3.1.

THEOREM 3.3. Let $\mathscr{H}$ be a saturated formation of full characteristic which is locally defined by the formation function $h$ given by $h(p)=\mathscr{I}_{\pi(p)}$, where $\pi(p)$ a set of primes with $p \in \pi(p)$, for all $p \in \wp$. The following statements are equivalent:

(i) $\mathscr{H}$ is a dominant Fitting class,

(ii) If $q \in \pi(p)$, then either $\pi(q)=\pi(p), \pi(q)=\wp$ or $\pi(p)=\wp$, for every $p \in \wp$.

REMARKS. (1) The construction of nilpotent injectors, Lockett injectors [6] and $p$-nilpotent injectors are particular cases of our general construction.

(a) Nilpotent injectors: Notice that $\mathscr{N}=L F(f)$ where $f(p)=\mathscr{S}_{\pi(p)}$ for $\pi(p)=$ $\{p\}$ and all $p \in \wp$. In this case, for a group $G$ and every prime $s \in \pi(G)$, we have 
$Z_{s}^{*}=C_{G}\left(O_{s^{\prime}}(F(G))\right)$. Given a Hall system $\Sigma$ of $G, H_{s}=G_{s} \cap Z_{s}^{*} \in \operatorname{Syl}_{s}\left(Z_{s}^{*}\right)$, where $G_{s} \in \Sigma \cap \operatorname{Syl}_{s}(G)$, and $H_{\Sigma}=\prod_{s \in \pi(G)} H_{s}$ is a nilpotent injector of $G$. Moreover, $\operatorname{Inj}_{\mathscr{N}}(G)=\left\{H_{\Sigma} \mid \Sigma\right.$ is a Hall System of $\left.G\right\}$.

(b) Lockett injectors [6]: Take $\left\{\pi_{i}\right\}_{i \in I}$ to be a family of pairwise disjoint sets of primes such that $\cup_{i \in I} \pi_{i}=\wp$. Let $\mathscr{F}=L F(f)$ where $f(p)=\mathscr{S}_{\pi_{i}}$ if $p \in \pi_{i}, i \in I$, for every prime $p \in \wp$. In this case $\pi(p)=\pi_{i}$, for all $p \in \pi_{i}$ and every $i \in I$. For a group $G$ and every prime $s \in \pi(G), Z_{s}^{*}=C_{G}\left(O_{\pi_{k}^{\prime}}(F(G))\right)$ if $s \in \pi_{k}, k \in I$. If $\Sigma$ is a Hall system of $G, H_{s}=G_{s} \cap Z_{s}^{*} \in \operatorname{Syl}_{s}\left(Z_{s}^{*}\right)$, where $G_{s} \in \Sigma \cap \operatorname{Syl}_{s}(G)$. Consequently $\prod_{s \in \pi_{k}} H_{s} \in \operatorname{Hall}_{\pi_{k}}\left(Z_{s}^{*}\right)$. So $H_{\Sigma}=\prod_{s \in \pi(G)} H_{s}=\prod_{k \in J}\left(\prod_{s \in \pi_{k}} H_{s}\right)$ is an injector of $G$. Again $\operatorname{Inj}_{\mathscr{F}}(G)=\left\{H_{\Sigma} \mid \Sigma\right.$ is a Hall System of $\left.G\right\}$.

(c) $p$-nilpotent injectors: $\mathscr{S}_{p}, \mathscr{S}_{p}=L F(f)$ where $f(p)=\mathscr{S}_{p}=\mathscr{S}_{\pi(p)}$ and $f(q)=$ $\mathscr{S}$ for every $q \in \wp \backslash\{p\}$, that is $\pi(q)=\wp$ if $q \in \wp \backslash\{p\}$. For a group $G, Z_{p}^{*}=G$ and $Z_{q}^{*}=C_{G}\left(O_{p^{\prime} p}(G)\right) \leq O_{p^{\prime} p}(G)$, if $q \neq p$. For a Hall system $\Sigma$ of $G, H_{p}=$ $G_{p}=G_{p} \cap Z_{p}^{*}$ where $G_{p} \in \Sigma \cap \operatorname{Syl}_{p}(G)$, and $H_{q}=G_{q} \cap Z_{q}^{*} \leq O_{p^{\prime} p}(G)$, if $q \neq p$, where $G_{q} \in \Sigma \cap \operatorname{Syl}_{q}(G)$. Therefore $H_{\Sigma}=O_{p^{\prime} p}(G) G_{p}=O_{p^{\prime}}(G) G_{p}$ is a $p$-nilpotent injector of $G$.

(2) If $\mathscr{F}=\mathscr{N}$ the following is true: For a group $G$ and every prime $s \in \pi(G)$ and each $H_{s} \in \operatorname{Syl}_{s}\left(Z_{s}^{*}\right)$, we have $\prod_{s \in \pi(G)} H_{s}$ as a nilpotent injector of $G$. Analogously, if $\mathscr{F}$ is a Fitting formation as in (b) and with the same notation, for a group $G$ and every set of primes $\pi_{i}, i \in I$, for each $H_{\pi_{i}} \in \operatorname{Hall}_{\pi_{i}}\left(Z_{s}^{*}\right), s \in \pi_{i}$, we have $\prod_{i \in l} H_{\pi_{i}}$ as an $\mathscr{F}$-injector of $G$. This is not true in general.

For instance take $\mathscr{F}=L F(f)$ where $f(p)=\mathscr{S}_{\pi(p)}$ for every prime $p \in \wp$, $\pi(3)=\{2,3\}, \pi(5)=\{5\}$ and $\pi(q)=\wp$, for every $q \notin\{3,5\}$. Let $V_{5}$ be an irreducible and faithful $\Sigma_{3}=\left[C_{3}\right] C_{2}$-module over $G F(5)$ and $V_{3}$ an irreducible and faithful $\left[V_{5}\right] \Sigma_{3}$-module over $G F(3)$. Construct $G=\left[V_{3}\right]\left(\left[V_{5}\right] \Sigma_{3}\right)$. For this group $G, Z_{2}^{*}=G, Z_{3}^{*}=G$ and $Z_{5}^{*}=C_{G}\left(V_{3}\right)=V_{3}$. We can take $G_{2} \in \operatorname{Syl}_{2}(G)$ and $G_{3} \in \operatorname{Syl}_{3}(G)$ such that $G_{2} G_{3} \neq G_{3} G_{2}$. So $G_{2} G_{3}$ cannot be an $\mathscr{F}$-injector of $G$. In fact, $\operatorname{Inj}_{\mathscr{F}}(G)=\left\{G_{2} G_{3} \mid G_{2} G_{3}=G_{3} G_{2}, G_{2} \in \operatorname{Syl}_{2}(G), G_{3} \in \operatorname{Syl}_{3}(G)\right\}$.

\section{Acknowledgement}

This research has been supported by Proyecto PB 94-0965 of DGICYT, Ministerio de Educación y Ciencia of Spain.

\section{References}

[1] A. Ballester-Bolinches, 'A note on saturated formations', Arch. Math. 58 (1992), 110-113.

[2] A. Ballester-Bolinches, K. Doerk and M. D. Pérez-Ramos, 'On the lattice of $\mathscr{F}$-subnormal subgroups', J. Algebra 148 (1992), 42-52. 
[3] A. Ballester-Bolinches, M. C. Pedraza-Aguilera and M. D. Pérez-Ramos, 'On $\mathscr{F}$-subnormal subgroups and $\mathscr{F}$-residuals of finite soluble groups', J. Algebra 186 (1996), 314-322.

[4] A. Ballester-Bolinches and M. D. Pérez-Ramos, 'On $\mathscr{F}$-critical groups', J. Algebra 174 (1995), 948-958.

[5] K. Doerk and T. Hawkes, Finite soluble groups (Walter De Gruyter, Berlin, 1992).

[6] F. P. Lockett, On the theory of Fitting classes of finite soluble groups (Ph.D. thesis, University of Warwick, 1971).

Departament d'Algebra

Universitat de València

C/- Doctor Moliner 50

46100 Burjassot (València)

Spain

e-mail: adolfo@uv.es

Departament d'Algebra

Universitat de València

C/- Doctor Moliner 50

46100 Burjassot (València)

Spain

e-mail: dolores.perez@uv.es
E. U. Informàtica

Departament de Matemàtica Aplicada Universitat Politècnica de València Camino de Vera s/n, 46071 València

Spain e-mail: anamarti@mat.upv.es 